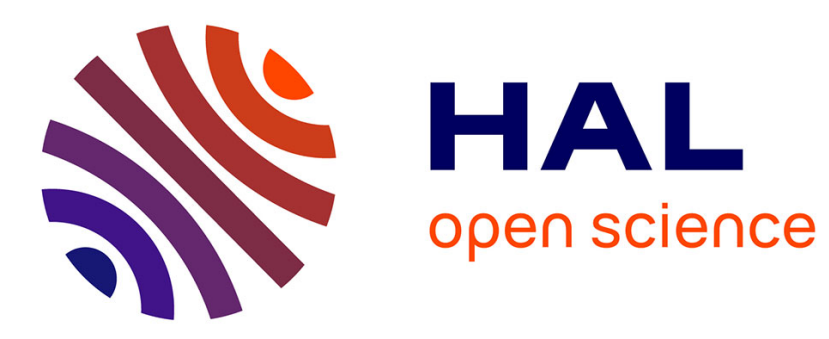

\title{
Impact of unresolved smaller scales on the scalar dissipation rate in direct numerical simulations of wall bounded flows
}

Cédric Flageul, Iztok Tiselj

\section{- To cite this version:}

Cédric Flageul, Iztok Tiselj. Impact of unresolved smaller scales on the scalar dissipation rate in direct numerical simulations of wall bounded flows. International Journal of Heat and Fluid Flow, 2017, 68, pp.173-179. 10.1016/j.ijheatfluidflow.2017.10.009 . hal-01631517

\section{HAL Id: hal-01631517 https://hal.science/hal-01631517}

Submitted on 9 Nov 2017

HAL is a multi-disciplinary open access archive for the deposit and dissemination of scientific research documents, whether they are published or not. The documents may come from teaching and research institutions in France or abroad, or from public or private research centers.
L'archive ouverte pluridisciplinaire HAL, est destinée au dépôt et à la diffusion de documents scientifiques de niveau recherche, publiés ou non, émanant des établissements d'enseignement et de recherche français ou étrangers, des laboratoires publics ou privés. 


\title{
Impact of unresolved smaller scales on the scalar dissipation rate in direct numerical simulations of wall bounded flows
}

\author{
Cédric Flageul, Iztok Tiselj \\ Reactor Engineering Division, Jožef Stefan Institut, Ljubljana, Slovenia
}

\begin{abstract}
Passive scalar dynamics in a turbulent channel flow is studied with Direct Numerical Simulation at friction Reynolds number $R e_{\tau}=160$ and Prandtl number $\operatorname{Pr}=1$. The goal of the study is to assess the grid spacing requirement for an accurate estimation of various integral turbulent statistics, with a special focus on the scalar dissipation rate. The implemented spatial resolutions span from the resolution comparable to the similar Direct Numerical Simulations (DNS) studies in the past, to the very fine resolution implemented by Galantucci and Quadrio [1]. All scalar fields are computed in parallel using a single velocity field resolved with the finest resolution, thus reducing the statistical variability. In addition, to confidently assess the grid spacing requirement, we also evaluate the statistical uncertainty. The "standard" resolution of the DNS studies (resolution used by Kim et al. [2]) is usually sufficient for predictions of first and second-order integral turbulence scalar field statistics. Non-negligible corrections of the fourth-order integral statistics, especially the scalar dissipation variance profile, are observed with enhancement of the scalar resolution from the one used in the "standard" DNS studies to the resolution recommended by Vreman and Kuerten [3], which is roughly two times finer in each spatial direction. Further resolution enhancements produce only marginal differences. Keywords: Passive scalar, Dissipation rate, Anisotropy, Direct Numerical Simulation, Channel flow, Sampling error
\end{abstract}




\section{Introduction}

Since the pioneering efforts of Obukhov [4, Corrsin [5] and Batchelor et al. (6. 7), passive scalars in turbulent flows have been the focus of a number of studies. As summarized in the review by Warhaft [8], experiments and simu-

5 lations are challenging classical descriptions of passive scalars derived from the Kolmogorov cascade phenomenology. Evidences suggest a strong coupling between large and small scales and no local isotropy at inertial and dissipation scales. They also suggest that passive scalars are associated with a stronger intermittency compared with the velocity.

Following Batchelor et al. ([6, 7]), for a unit Prandtl number as herein, the smallest spatial scale for scalar mixing is the Kolmogorov scale $\eta=\left(\nu^{3} / \bar{\varepsilon}\right)^{1 / 4}$, where $\nu$ is the dynamic viscosity and $\bar{\varepsilon}$ the mean dissipation rate of the turbulent kinetic energy. Similarly, the smallest time scale is the Kolmogorov time scale $\tau_{\eta}=\sqrt{\nu / \bar{\varepsilon}}$. As the passive scalar is intermittent, locally, structures with a 15 spatial (temporal) span shorter than $\eta\left(\tau_{\eta}\right)$ appear in a flow. Suspecting those fine structures to be related to highly dissipative events, a number of DNS have been performed with sub-Kolmogorov scales resolved (Schumacher et al. 9], Donzis and Yeung [10], Galantucci and Quadrio [1]).

One of the central quantity in those studies is $\varepsilon_{\theta}$, the scalar dissipation rate, defined by

$$
\varepsilon_{\theta}=2 \alpha|\nabla \theta|^{2}=2 \alpha\left[\sum_{i=1}^{3}\left(\partial_{i} \theta\right)^{2}\right]
$$

where $\alpha$ is the thermal diffusivity. According to Pope [11, this quantity he calls the all-important dissipation rate matters in combustion models. It is also a central quantity in Reynolds Averaged Navier-Stokes (RANS) turbulence models as it appears in the budget equation of the scalar variance. Lately, Flageul et al. [12] showed that the dissipation rate associated with the temperature variance is discontinuous at the fluid-solid interface in case of conjugate heat transfer. This is prominent for industrial applications where thermal fatigue is a concern. 
Studying homogeneous isotropic turbulence, Schumacher et al. 9] showed that the improved resolution matters when investigating the tails of the Probability Density Function (PDF) of $\varepsilon_{\theta}$, which correspond to low probability events associated with high or low dissipation rates. More specifically, they show that a poorer resolution has a stronger impact on regions of low $\varepsilon_{\theta}$ than on those of high $\varepsilon_{\theta}$. On a similar configuration, Donzis and Yeung [10] showed accurate estimation of advanced statistics (scalar dissipation intermittency exponent, structure functions at moderately high orders and PDF of $\varepsilon_{\theta}$ up to $200 \overline{\varepsilon_{\theta}}$ ) with a grid spacing equal to the Batchelor scale, which is exactly the Kolmogorov scale in the present study.

Studying a turbulent channel flow $\left(R e_{\tau}=160, \operatorname{Pr}=1\right)$, Galantucci and Quadrio [1] extended the analysis to wall-bounded flows. They showed resolution effects on the profiles of the mean $\varepsilon_{\theta}$ and its variance, but also on the

40 $\mathrm{PDF}$ of $\varepsilon_{\theta}$ and recommended a very fine spatial resolution $\left(\Delta_{x}^{+}=\Delta_{z}^{+}=1\right.$ and $0.43<\Delta_{y}^{+}<2$ ). In the streamwise (spanwise) direction, this is 6 (4) times finer than what is necessary for the velocity according to Vreman and Kuerten [3. The authors of the present study estimate that the resolution proposed by Vreman and Kuerten is sufficient for accurate predictions of the key integral turbulent statistics of the passive scalar field at $\operatorname{Pr}=1$, including the average scalar dissipation rate and its variance. The main objective of the present paper is to assess this claim.

The structure of the paper is as follows. In the second section, the governing equations and the computational setup are described alongside with the procedure to estimate the sampling error. In the third section, preliminary investigation on coarser grids is presented. In the fourth section, the DNS results are presented. Discussion and conclusions are collected in the last section.

\section{Governing equations, computational setup and sampling error}

Dimensionless equations of the incompressible turbulent channel flow with transport of a passive scalar can be found in various sources (Kasagi et al. [13], 
Kawamura et al. [14]):

$$
\begin{aligned}
& \nabla \cdot u=0 \\
& \partial_{t} u=-\nabla \cdot(u \cdot u)+\frac{1}{R e_{\tau}} \nabla^{2} u-\nabla(p)+\overrightarrow{1_{x}} \\
& \partial_{t} \theta=-\nabla \cdot(u \cdot \theta)+\frac{1}{R e_{\tau} P r} \nabla^{2} \theta
\end{aligned}
$$

Equations (3) and (4) are normalized with the channel half width $h$, the kinematic viscosity $\nu$ and the friction velocity $u_{\tau}$. Low friction Reynolds number $R e_{\tau}=160$ and Prandtl number $\operatorname{Pr}=1$ were selected in order to replicate the conditions of the simulations performed by Galantucci and Quadrio [1. As the Prandtl number is unity, Kolmogorov and Batchelor length-scales are identical.

Periodic boundary conditions are used in the streamwise and spanwise directions, labelled $x$ and $z$, respectively, while the wall-normal direction is labelled $y$. The forcing term $\overrightarrow{1_{x}}$ represents a constant pressure gradient in the streamwise direction and has a unit amplitude thanks to the normalization used. Boundary conditions for the passive scalar fields at the channel walls are $\theta=1$ at $y=1$, and $\theta=-1$ at $y=-1$ and were previously used in the simulations of Papavassiliou and Hanratty [15, Johansson and Wikström [16, and Galantucci and Quadrio [1].

The equations are solved with a pseudo-spectral scheme. Fourier series are used in the $x$ and $z$ directions and Chebyshev polynomials are used in the $y$ direction. Second-order accurate time differencing (Crank-Nicolson scheme for diffusive terms and Adams-Bashforth scheme for other terms) is used with maximum Courant number kept at approximately 0.1. The aliasing error is removed with computation of the nonlinear terms on a grid 1.5 times finer in all directions. The computer code is based on the code developed by Gavrilakis et al. [17, which was later modified by Lam and Banerjee [18. The code was used and verified in simulations of Tiselj et al. ([19, [20, 21]).

The extension of the computational domain, normalized with the channel so half width $h$, was taken from the work of Galantucci and Quadrio [1]: $L_{x}=4.19$, 


\begin{tabular}{|c|c|c|c|c|}
\hline Scalar field & $N x * N y * N z$ & $\Delta_{x}^{+}$ & {$\left[\Delta_{y, \text { min }}^{+}, \Delta_{y, \text { max }}^{+}\right]$} & $\Delta_{z}^{+}$ \\
\hline $1 \mathrm{KMM}$ & $40 * 129 * 60$ & 16.8 & {$[0.048,3.93]$} & 5.57 \\
\hline $2 \mathrm{GQC}$ & $64 * 129 * 64$ & 10.5 & {$[0.048,3.93]$} & 5.23 \\
\hline $3 \mathrm{~S}$ & $112 * 129 * 80$ & 5.99 & {$[0.048,3.93]$} & 4.18 \\
\hline $4 \mathrm{VK}$ & $112 * 181 * 80$ & 5.99 & {$[0.024,2.78]$} & 4.18 \\
\hline $5 \mathrm{GQM}$ & $360 * 129 * 180$ & 1.86 & {$[0.048,3.93]$} & 1.86 \\
\hline $6 \mathrm{~S}$ & $360 * 181 * 180$ & 1.86 & {$[0.024,2.78]$} & 1.86 \\
\hline
\end{tabular}

Table 1: Spatial resolution for the transported passive scalar fields.

$L_{y}=2$, and $L_{z}=2.09$. Both in the streamwise and spanwise directions, this is about 3 times smaller than the domain used in Kasagi et al. 13. Such a small computational domain neglects an important part of the large scale structures in the turbulent flow, however, it is known to be sufficient for special studies focused on small scale turbulent structures of the velocity field (Jimenez and Moin 222]). The small domain offers a platform for simplified studies of the resolution requirements. It is often overlooked that in addition to obliterating large scale structures, small domain can be affected by a significant sampling error. For instance, Galantucci and Quadrio [1] report up to $5 \%$ differences in the friction temperature values in their simulations performed on different resolutions and averaged over the time interval of 2400 viscous time units (statistics based on 60 instantaneous fields).

In the present study, the resolution requirement for the passive scalar field is examined with a single DNS run. The velocity field is calculated on the finest grid of $N x * N y * N z=360 * 181 * 180$ modes (Case $6 \mathrm{~S}$ in Table1). Six distinct passive scalar fields are simultaneously transported by this velocity field and resolved with different number of modes, see Table 1 . The naming scheme for the passive scalar fields is inspired by the name of the authors who promoted certain resolution. For instance, in 1KMM, KMM stands for Kim, Moser and Moin 2. Similarly, in 4VK, VK stands for Vreman and Kuerten [3]. The number preceding the letters corresponds to a disputable ranking of the grids: 
1 for the coarsest, 6 for the finest. For the five scalar fields resolved with a lower resolution, all Fourier and Chebyshev modes above the indicated resolution are set to zero at the end of each time step.

This approach, which is comparable to the one in Brethouwer et al. 23 or Gotoh et al. 24, reduces the statistical variability and eases the separation of the sampling error from the error induced by a coarser spatial resolution. The separation of statistical uncertainty and resolution effects is of particular importance for the present work: the smaller extension of the domain in the homogeneous directions increases the sampling error, which easily exceeds the tiny differences induced by the variable resolution of the passive scalar field, except for simulations with a very long duration.

The finer resolution (case $6 \mathrm{~S}$ in Table 1) used in the wall-normal direction follows Vreman and Kuerten [3]. In the streamwise and spanwise directions, case $6 \mathrm{~S}$ corresponds to the resolution used by Galantucci and Quadrio [1] in their "Medium" simulation. This is 2 to 3 times finer than the recommendation of Vreman and Kuerten [3. Cases 5GQM and 2GQC correspond to the "Medium" and "Coarse" cases in Galantucci and Quadrio [1, respectively. The case 4VK is using the resolution recommended by Vreman and Kuerten [3]. The case $3 \mathrm{~S}$ is similar to $4 \mathrm{VK}$ except that it uses a coarser wall-normal grid. Lastly, the resolution in case $1 \mathrm{KMM}$ is comparable with most of the previous DNS simulations (Kim et. al. [2, Kasagi et al. [13, Tiselj et al. [19]).

The present DNS is performed with a time step of $0.008 \nu / u_{\tau}^{2}$ and one snapshot is taken every 1000 time steps. 700 snapshots are used to reconstruct the statistics, corresponding to an averaging time of $5600 \nu / u_{\tau}^{2}$.

DNS is widely used to produce reference data. However, as pointed out by Oliver et al. 25], statistics obtained from DNS contain non-trivial errors. Errors arise mainly from the discretization of the equations and from the finite statistical sampling. As our code is based on a pseudo-spectral method and we use a fine grid, the spatial discretization error of the Navier-Stokes equation is rather low. Thus, the main source of error in our statistics is the sampling frror, which we estimate with the open-source library ar https://github. 
com/RhysU/ar, see Oliver et al. 25]).

The program arsel, which ships with the library, is used as follows. For each statistical quantity of interest, the instantaneous value is extracted from each snapshot and averaged in the homogeneous directions. The resulting time series are used to fit autoregressive models with arsel using the --subtract-mean option. The output of arsel is then processed to extract the average profile and the sampling error profile. Herein, the sampling error is given as 2 standard deviations. Given the domain extension, the number of modes used and the time step, the probability that the real average and its estimation differ for less than 2 standard deviations is $95 \%$.

As this method can be applied for any statistical quantity, we are able to estimate the sampling error for the mean temperature, the temperature dissipation rate or its variance. For a given variable $\phi$, the associated sampling error is $e_{\phi}$. We also define the dimensionless normalized sampling error $E[\phi]$ by

$$
E[\phi](y)=\frac{e_{\phi}(y)}{e_{\phi}(y)+\operatorname{abs}(\overline{\phi(y)})}
$$

This normalized sampling error is bounded in $[0,1]$, the upper bound being reached when the averaged value abs $(\overline{\phi(y)})$ is exactly zero. As most of the time the averaged value is large compared to the sampling error, $E[\phi]$ measures the relative uncertainty.

\section{Preliminary results on coarser grids}

The sampling error on $u_{\tau}^{2}$, whose theoretical value is 1 herein, and $\partial_{y} \bar{\theta}(y= \pm 1)$ is illustrated with the four DNS studies on coarser grids described in the Table 2. The results in the large computational box combined with the long and short averaging time are taken from the analyses of Tiselj and Cizelj [20], which were performed at $R e_{\tau}=180, \operatorname{Pr}=1$ and with slightly different thermal boundary conditions: both channel walls were heated and a forcing term was present in the passive scalar equation (4). The small box results are part of the present anal155 ysis: a separate run over longer time interval was made only with a resolution, 


\begin{tabular}{|c|c|c|c|c|c|c|}
\hline Label & $R e_{\tau}$ & $\begin{array}{c}L_{x}^{+} \\
L_{y}^{+} \\
L_{z}^{+} \\
\end{array}$ & $\begin{array}{c}\Delta_{x}^{+} \\
\Delta_{y}^{+}{ }_{, \max }^{+} \\
\Delta_{z}^{+}\end{array}$ & $\begin{array}{l}\text { Averaging time } \\
\text { in wall units }\end{array}$ & $u_{\tau}^{2} \pm e_{u_{\tau}^{2}}$ & $\partial_{y} \bar{\theta} \pm e_{\partial_{y} \bar{\theta}}$ \\
\hline $18 \mathrm{~L}$ & \multirow{2}{*}{180} & \multirow{2}{*}{$\begin{array}{c}6786 \\
360 \\
2262\end{array}$} & \multirow{2}{*}{$\begin{array}{l}17.7 \\
4.42 \\
5.89\end{array}$} & $\begin{array}{c}24300 \\
\text { (450 samples) }\end{array}$ & $\begin{array}{l}1.0004 \pm 0.0016 \\
0.9998 \pm 0.0016\end{array}$ & $\begin{array}{c}-180.11 \pm 0.15 \\
180.01 \pm 0.14\end{array}$ \\
\hline $18 \mathrm{~S}$ & & & & $\begin{array}{c}5400 \\
(100 \text { samples })\end{array}$ & $\begin{array}{l}0.9997 \pm 0.0029 \\
1.0005 \pm 0.0033\end{array}$ & $\begin{array}{c}-179.74 \pm 0.29 \\
180.14 \pm 0.46\end{array}$ \\
\hline $16 \mathrm{~L}$ & \multirow{2}{*}{160} & \multirow{2}{*}{$\begin{array}{l}670 \\
320 \\
334\end{array}$} & \multirow{2}{*}{$\begin{array}{l}16.8 \\
3.93 \\
5.57\end{array}$} & $\begin{array}{c}24000 \\
\text { (500 samples) }\end{array}$ & $\begin{array}{l}1.002 \pm 0.014 \\
1.003 \pm 0.019\end{array}$ & $\begin{array}{l}6.80 \pm 0.05 \\
6.78 \pm 0.08\end{array}$ \\
\hline $16 \mathrm{~S}$ & & & & $\begin{array}{c}6000 \\
(125 \text { samples })\end{array}$ & $\begin{array}{l}1.005 \pm 0.026 \\
0.990 \pm 0.050\end{array}$ & $\begin{array}{l}6.82 \pm 0.10 \\
6.63 \pm 0.24\end{array}$ \\
\hline
\end{tabular}

Table 2: Sampling error on friction velocity and temperature derivative at the boundaries.

which corresponds to the case 1KMM in Table 1

The first impression about the statistical uncertainty can be obtained from the two rightmost columns of Table 2 where the squared friction velocities and temperature derivatives at both channel walls are shown. As one might expect, deviation from the theoretical friction velocity of 1 is larger in the smaller computational domain and at shorter averaging time intervals. As for the temperature derivative, shorter averaging time also leads to higher sampling error. Regarding cases $R e_{\tau}=160$, we observe a relatively large difference on the temperature derivative at both walls. Although this difference gets lower when increasing the duration of the simulation, it is probably specific to smaller computational domains, as reported by Jimenez and Moin 22] for velocity statistics.

The case $16 \mathrm{~S}$ in Table 2 is comparable to the case $1 \mathrm{KMM}$ in Table 1. Both cases correspond to an averaging time roughly 2 times longer than the one in Galantucci and Quadrio [1], with snapshots taken at a similar frequency for all those cases. Thus, the case $16 \mathrm{~S}$ gives a lower bound for the sampling error in Galantucci and Quadrio [1. The Figure 1 shows the normalized sampling error associated with various statistical quantities for the cases present in Table 2 . 

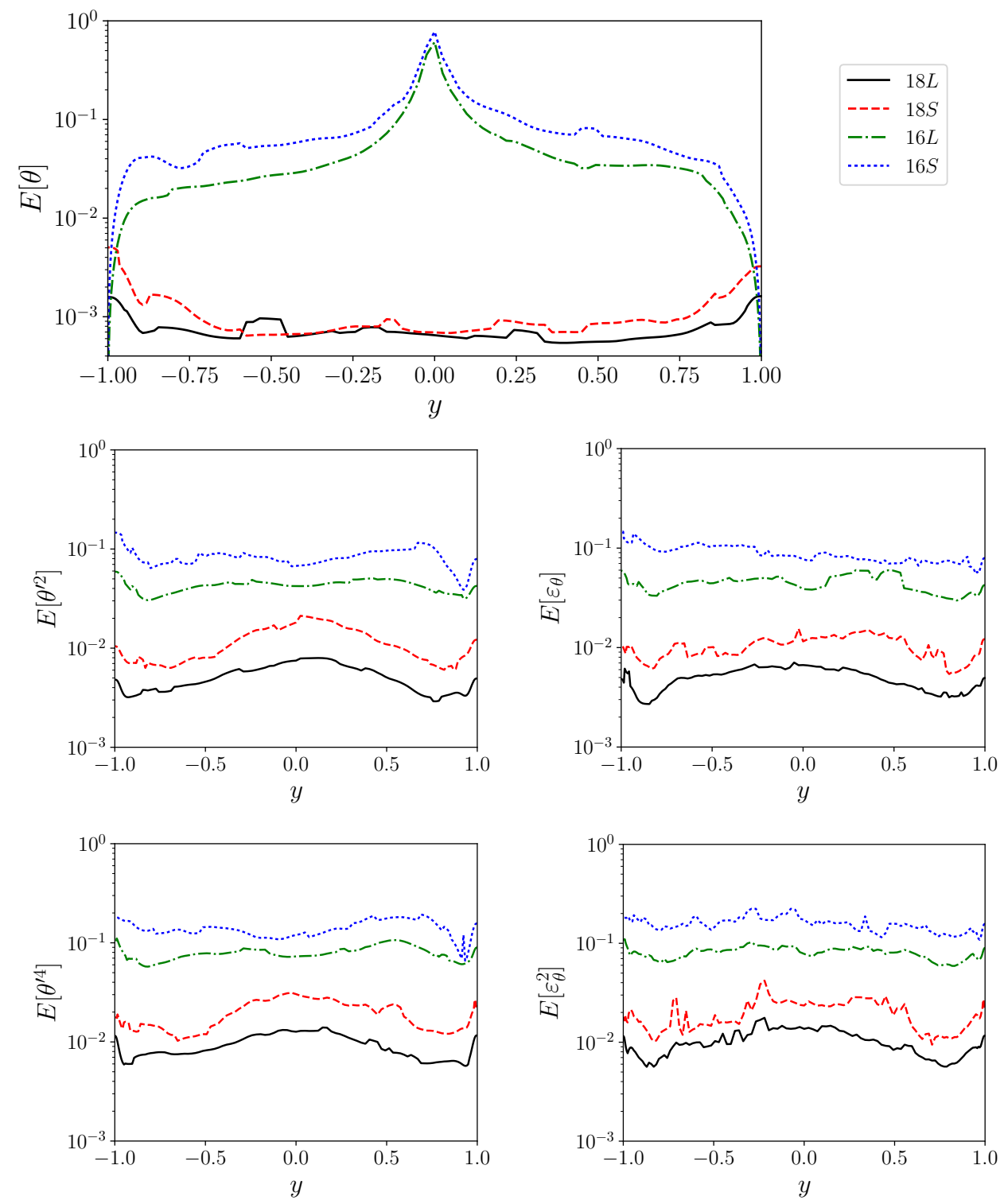

Figure 1: Normalized sampling error for the cases in Table 2 Top: $E[\theta]$. Middle: $E\left[\theta^{\prime 2}\right]$ (left) and $E\left[\varepsilon_{\theta}\right]$ (right). Bottom: $E\left[\theta^{\prime 4}\right]$ (left) and $E\left[\varepsilon_{\theta}^{2}\right]$ (right). 
The clear trend in Figure 1 is that higher order moments are associated with higher sampling errors. Regarding the plots on the middle row, the normalized sampling error for the temperature variance and for the mean dissipation rate have a similar amplitude and involve turbulent quantities to the power 2. Qualitatively, the situation is similar on the plots in the bottom row, which involve turbulent quantities to the power 4.

Regarding case 16S, the magnitude of the normalized sampling error is around $5 \%$ for $E[\theta]$, except in the middle of the channel, where it gets close to $100 \%$ as the average temperature is zero. It also drops down to zero at the walls as the temperature is imposed there (Dirichlet boundary condition, $\theta= \pm 1$ ). For cases $18 \mathrm{~S}$ and $18 \mathrm{~L}$, the imposed temperature at the walls is zero, so both the sampling error and the average temperature tend to zero there. The resulting normalized sampling error $E[\theta]$ at the wall for those cases is around $0.2 \%$.

Focusing on case $16 \mathrm{~S}$ for higher order integral statistics, the normalized sampling error reaches almost $10 \%$ for $E\left[\theta^{\prime 2}\right]$ and $E\left[\varepsilon_{\theta}\right]$, and even $15 \%$ for $E\left[\theta^{\prime 4}\right]$ and $E\left[\varepsilon_{\theta}^{2}\right]$. These results show that the differences of $7 \%$ on the average of $\varepsilon_{\theta}$ on different grid reported in Galantucci and Quadrio [1] is well within the sampling error as their simulations have the same domain extension but a duration halved compared with the case 16S.

Using the Central Limit Theorem applied to weakly correlated samples described in Oliver et al. 25] (Theorem 27.4 in Billingsley [26]), the sampling error decreases with the square root of the number of samples. For a given sampling 195 frequency, the error is therefore halved when the duration of the simulation (and the number of samples) is increased by a factor 4 . This holds quite well when comparing cases $16 \mathrm{~S}$ and $16 \mathrm{~L}$, or $18 \mathrm{~S}$ and $18 \mathrm{~L}$. Overall, significant reduction of the sampling error can be achieved using extended computational domains and / or simulations with a longer duration. 


\begin{tabular}{|c|c|c|c|c|c|c|c|}
\hline Scalar field & $N x * N y * N z$ & $\theta$ & $\theta^{\prime 2}$ & $\theta^{\prime 3}$ & $\theta^{\prime 4}$ & $\varepsilon_{\theta}$ & $\varepsilon_{\theta}^{\prime 2}$ \\
\hline $1 \mathrm{KMM}$ & $40 * 129 * 60$ & -7.18 & -5.02 & -4.42 & -3.67 & -6.68 & -1.26 \\
\hline $2 \mathrm{GQC}$ & $64 * 129 * 64$ & -9.15 & -7.14 & -6.50 & -5.43 & -7.82 & -1.91 \\
\hline $3 \mathrm{~S}$ & $112 * 129 * 80$ & -11.8 & -10.5 & -8.57 & -8.11 & -9.54 & -3.25 \\
\hline 4VK & $112 * 181 * 80$ & -11.8 & -10.5 & -8.58 & -8.13 & -9.98 & -3.29 \\
\hline $5 \mathrm{GQM}$ & $360 * 129 * 180$ & -13.9 & -12.0 & -10.0 & -10.8 & -9.51 & -5.53 \\
\hline
\end{tabular}

Table 3: $\log _{10}$ of the quadratic relative error for various quantities.

\section{Impact of unresolved smaller scales on statistics}

In this section, we investigate the impact of unresolved smaller scales on various quantities. First, we investigate dimensionless bulk quantities. Using the scalar with the finest resolution $(6 \mathrm{~S})$ as a reference, we can compute a quadratic relative error. For instance, the quadratic relative error for the average temperature on the grid $1 \mathrm{KMM}$ is defined by:

$$
\frac{\int_{y=-1}^{y=1}\left[\overline{\theta_{1 K M M}}(r)-\overline{\theta_{6 S}}(r)\right]^{2} \mathrm{dr}}{\int_{y=-1}^{y=1} \overline{\theta_{6 S}}(r)^{2} \mathrm{dr}}
$$

In Table 3 the quadratic relative error is given for various quantities. Looking at the relative error for the moments of the temperature $\left(\theta, \theta^{\prime 2}, \theta^{\prime 3}\right.$ and $\left.\theta^{\prime 4}\right)$, one can extract two trends. First, for all resolutions considered, the higher the moment, the higher the relative error. Second, the finer the resolution, the closer to the case $6 \mathrm{~S}$. The resolution $1 \mathrm{KMM}$ seems sufficient to accurately estimate the average temperature. However, accurate estimation of higher order moments requires a finer resolution as unresolved smaller scales have a higher impact on them. The situation is similar for $\varepsilon_{\theta}$ and $\varepsilon_{\theta}^{\prime 2}$ : the first is relatively well estimated by the coarsest grid while the second has a stronger dependence on unresolved smaller scales.

It is important to stress that the discretization error in Table 3 is typically several decades lower than the sampling error. However, all the scalar fields are transported by the same velocity field. Therefore, they are strongly correlated 
and are all plagued by the same sampling error. This allows us to exhibit the impact of unresolved smaller scales on statistics, even though this impact is relatively small compared with the sampling error.

For the turbulent channel flow studied here, at the wall, the temperature is imposed $(\theta= \pm 1)$ and the temperature evolution equation 4 contains no source term. As a result, the exact average temperature derivative at the wall is plagued by statistical uncertainty and depends on the spatial resolution. We define the friction temperature $\theta_{\tau}$ and the reference temperature $\theta_{\text {ref }}$ with:

$$
\theta_{\tau}=\frac{1}{R e_{\tau} \operatorname{Pr}} \partial_{y} \bar{\theta}\left( \pm \frac{L_{y}}{2}\right) \text { and } \theta_{r e f}=\frac{1}{R e_{\tau} \operatorname{Pr}} \frac{\bar{\theta}\left(L_{y} / 2\right)-\bar{\theta}\left(-L_{y} / 2\right)}{L_{y}}
$$

The theoretical value of the friction velocity, which is equal to one here, produces a $\theta_{\text {ref }}$ not plagued by statistical uncertainty, while $\theta_{\tau}$ is. Therefore, we use $\theta_{\text {ref }}$ to rescale the temperature statistics. Spatial derivatives are expressed in wall-units using the theoretical value of the friction velocity. For convenience, plots are using a wall-normal distance in computational units $(y \in[-1,1])$.

Complementary with the bulk quantities in Table 3 and the integral profiles of $\varepsilon_{\theta}$ or its variance, we investigate the anisotropy of the fluctuating temperature gradient. This is done using the barycentric map defined by Banerjee et al. 27. Given a symmetric positive semi-definite tensor $M_{i j}$, the associated (traceless) anisotropy tensor is

$$
\widetilde{M_{i j}}=\frac{M_{i j}}{M_{k k}}-\frac{1}{3} \delta_{i, j}
$$

The eigenvalues of $\widetilde{M_{i j}}, \lambda_{k}$, verify $\lambda_{1} \geq \lambda_{2} \geq \lambda_{3}$. Based on those eigenvalues, Banerjee et al. 27] define 3 limiting states forming a basis: one-component (1C), two-component $(2 \mathrm{C})$ and three-component $(3 \mathrm{C})$. The number of components corresponds to the number of non-zero eigenvalues of $M_{i j}$. In the state $3 \mathrm{C}$, all three eigenvalues are equal: the tensor is isotropic. In the state $2 \mathrm{C}$, one eigenvalue is negligible while the two others are equal: the anisotropy is twodimensional. In the state $1 \mathrm{C}$, two eigenvalues are negligible: the anisotropy is one-dimensional.

In the barycentric map, each limiting state is the vertex of an equilateral triangle. For any symmetric positive semi-definite tensor $M_{i j}$, there is a unique 
point inside this equilateral triangle with coordinates

$$
[x, y]=\left(\lambda_{1}-\lambda_{2}\right)\left[x_{1 C}, y_{1 C}\right]+2\left(\lambda_{2}-\lambda_{3}\right)\left[x_{2 C}, y_{2 C}\right]+\left(3 \lambda_{3}+1\right)\left[x_{3 C}, y_{3 C}\right]
$$
$3 \mathrm{C})$.

Focusing on the left frame of Figure 2, one may notice that the profiles of 

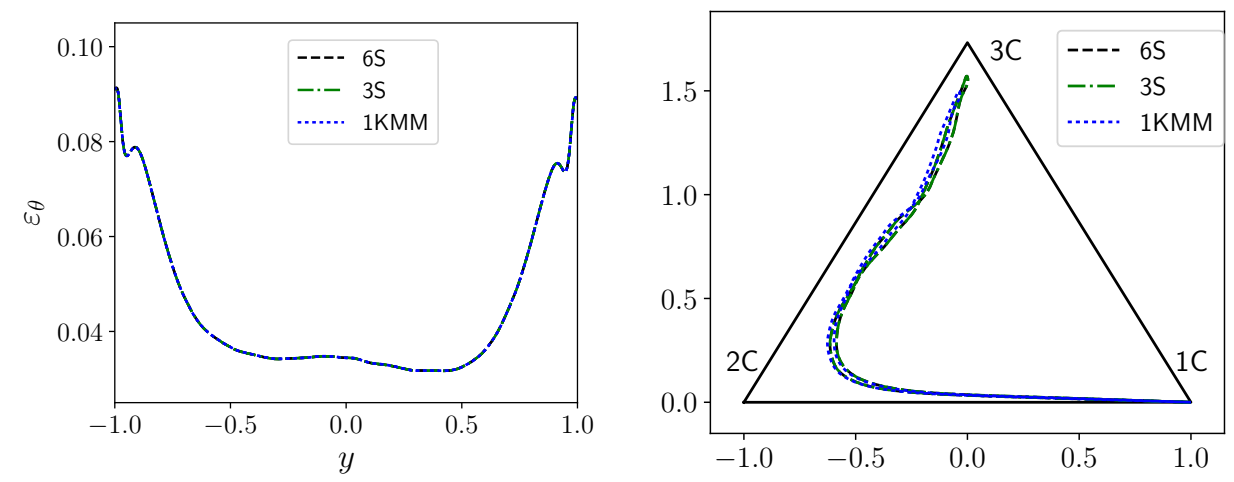

Figure 2: Dissipation rate $\overline{\varepsilon_{\theta}}$ (left) and barycentric map associated with $\overline{\overline{\partial \theta}}$ (right).

$\varepsilon_{\theta}$ are not exactly symetric. This is not an issue here as this is well within the sampling error, which is around $10 \%$ for $\varepsilon_{\theta}$, according to Figure 1 However, performing a simulation with a duration long enough to have a negligible sampling error, one may obtain a profile that still lacks symetry. This is specific to the combination of smaller domains and periodicity used here: global mixing between the lower and upper half of the domain is not perfect due to the absence of large scale structures.
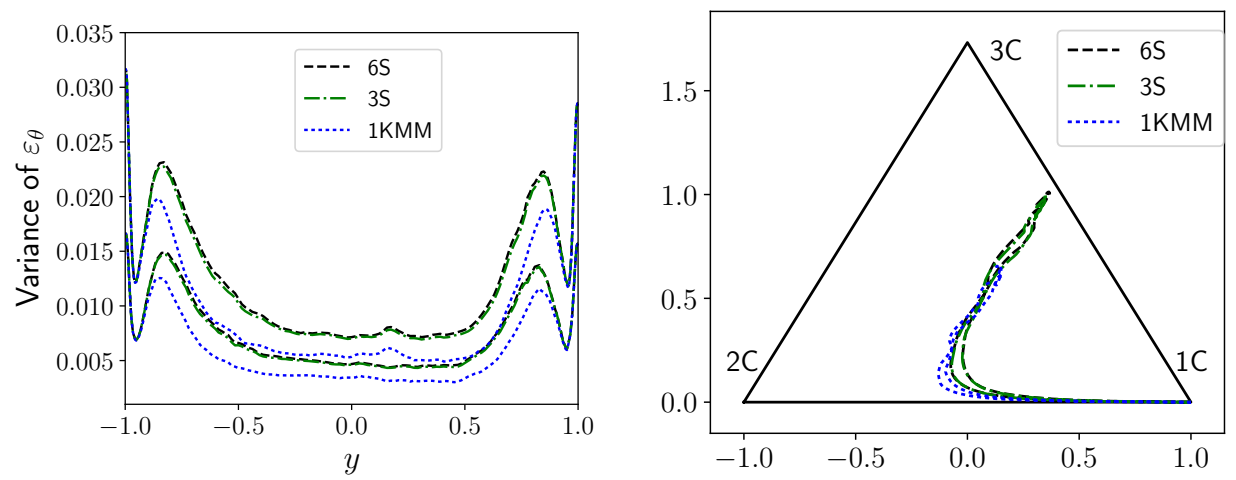

Figure 3: $95 \%$ confidence interval of the variance of the dissipation rate $\varepsilon_{\theta}$ (left) and barycentric map associated with $\sigma_{\overline{\overline{\partial \theta}}}$ (right). 

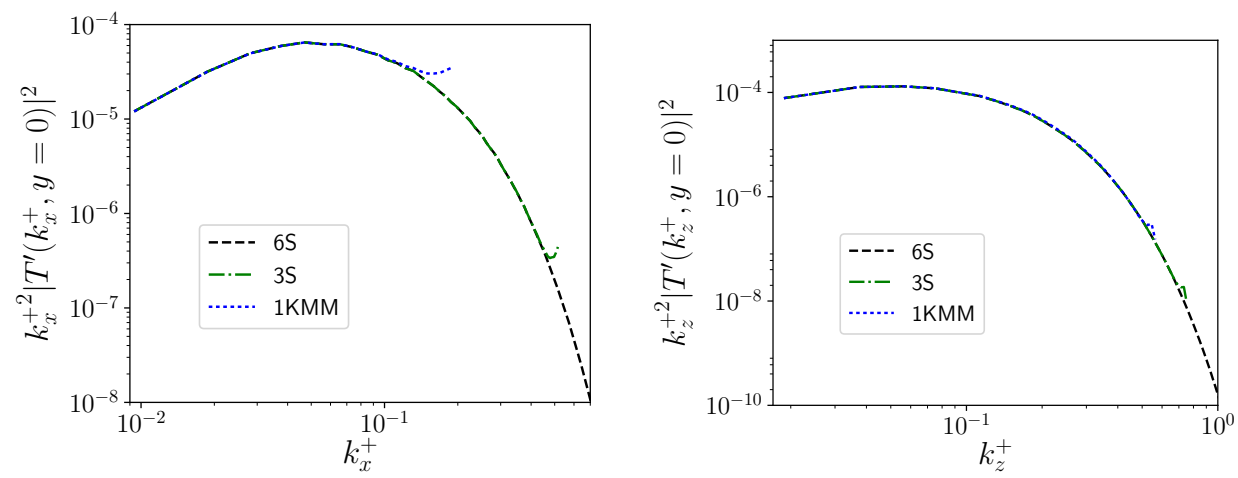

Figure 4: Spectra of the streamwise (left) and spanwise (right) contributions to the scalar dissipation rate $\varepsilon_{\theta}$ at $y=0$ (middle of the channel).

Integral quantities provide only a limited amount of information and spectra in Figure 4 are more insightful. They are taken at the middle of the channel $(y=0)$. The spanwise spectra show that the spanwise contribution to $\varepsilon_{\theta}$ is well resolved even on the coarsest grid. However, this grid is not isotropic and it has extended cells in the streamwise direction $\left(\Delta_{x}^{+}=17, \Delta_{z}^{+}=6\right.$ for $\left.1 \mathrm{KMM}\right)$. This leads to a pronounced bump on the streamwise spectrum of $1 \mathrm{KMM}$ at the highest resolved wavenumbers $\left(k_{x}^{+} \approx 0.2\right)$. As one can notice, the streamwise 
spectrum of $3 \mathrm{~S}$ also has a bump at the highest resolved frequency $\left(k_{x}^{+} \approx 0.55\right)$.

However, regarding the latter case, this has a negligible impact on the statistics of $\varepsilon_{\theta}$ because the bumps correspond to very high frequencies for which the streamwise contribution to the dissipation is two decades smaller than its peak value around $k_{x}^{+} \approx 0.05$. This does not hold for case $1 \mathrm{KMM}$ : the bump on the streamwise spectrum is present at lower frequencies, for which the streamwise contribution to the dissipation has a magnitude similar to the one at the peak. This explains the severe impact of unresolved smaller scales on the variance of $\varepsilon_{\theta}$ exhibited in Figure 3
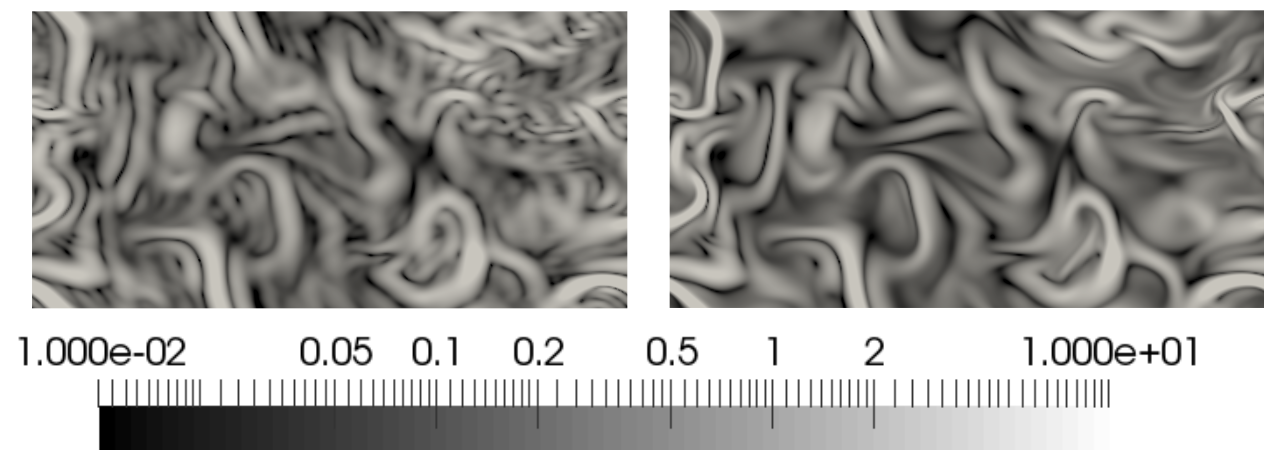

Figure 5: Instantaneous scalar gradient $\left(\sum \partial_{i} \theta^{\prime 2}\right)$ in computational units at $y=0$ (middle of the channel). Logarithmic colormap. Left: 1KMM. Right: 6S.

Figure 5 illustrates the impact of the under-resolution on the gradient of the scalar. On the one hand, both fields exhibit similar structures at larger scales, in agreement with the ability of the grid 1KMM to estimate the average scalar dissipation rate, as shown in Figure 2. On the other hand, smaller scales are visibly misrepresented on the coarser grid, as the picture on the left is plagued by the oscillations, which correspond to the wavenumbers of around $k^{+}=0.2$ that are overpredicted in $1 \mathrm{KMM}$ results shown in the left frame of Figure 4. This is consistent with the previous results: the grid 1KMM is too coarse to properly resolve the sharp gradients which separate dissipative and non-dissipative regions. 


\section{Conclusion}

The present investigation demonstrates the impact of a limited resolution

305 anisotropy of the fluctuating scalar gradient. In a preliminary part, we showed that simulations of the turbulent channel flow based on smaller domains and with a limited duration (often used to perform grid sensitivity analysis) are plagued by a relatively large sampling error, especially for higher order moments scalar dissipation rate with a relatively low discretization error, as indicated Table 3. However, they can only provide qualitative estimation for higher order moments. To accurately estimate those higher order moments, finer grids must be used. Regarding the variance of the scalar dissipation rate, or the and Kuerten [3] is sufficient for pseudo-spectral schemes $\left(6^{\text {th }}\right.$ order compact finite-difference schemes typically require a grid $4 / 3$ finer). Our results do not 
preclude existence of scalar structures or events, which are too small to be resolved with the Vreman and Kuerten resolution. However, our results show that these events might significantly affect only the integral turbulence statistics higher than fourth-order. It is important to stress that a small event can impact part of the PDF of a turbulent quantity without significantly affecting the associated integral value.

Our results also suggest that unresolved smaller scales have a stronger impact outside of the viscous sublayer, and have almost no impact closer to the wall. Strictly speaking, this observation is valid only for the Dirichlet boundary condition investigated here (imposed temperature), and may not hold for a Neumann boundary condition (imposed heat flux), a Robin boundary condition (heat exchange coefficient) or conjugate heat-transfer (fluid-solid thermal cou345 pling). Regarding the latter case, the present analysis will provide a solid ground for future investigations on the discontinuity of $\varepsilon_{\theta}$ at the fluid-solid interface.

口. Data associated with the present paper are available online at http://dx. doi.org/10.17632/mn74gv69wn.1 and/https://repo.ijs.si/CFLAG/sml-scl under the GNU GPL v3 licence.

\section{Acknowledgements}

This work was financially supported by the research project of the Slovenian Research Agency P2-0026 and by the EDF-JSI collaboration, project PR-07184.

\section{References}

[1] L. Galantucci, M. Quadrio, Very fine near-wall structures in turbulent scalar mixing, International Journal of Heat and Fluid Flow 31 (4) (2010) 499-506. doi:10.1016/j.ijheatfluidflow.2010.04.002

[2] J. Kim, P. Moin, R. Moser, Turbulence statistics in fully developed channel flow at low reynolds number, Journal of fluid mechanics 177 (1987) 133-166. doi:10.1017/S0022112087000892. 
[3] A. W. Vreman, J. G. M. Kuerten, Comparison of direct numerical simulation databases of turbulent channel flow at re $\tau=180$, Physics of Fluids 26 (1) (2014) 015102. doi:10.1063/1.4861064

[4] A. M. Obukhov, Structure of the temperature field in turbulent flow, Tech. rep., DTIC Document (1968).

[5] S. Corrsin, On the spectrum of isotropic temperature fluctuations in an isotropic turbulence, Journal of Applied Physics 22 (4) (1951) 469-473. doi:10.1063/1.1699986.

[6] G. K. Batchelor, Small-scale variation of convected quantities like temperature in turbulent fluid part 1. general discussion and the case of small conductivity, Journal of Fluid Mechanics 5 (01) (1959) 113-133. doi:10.1017/S002211205900009X.

[7] G. K. Batchelor, I. D. Howells, A. A. Townsend, Small-scale variation of convected quantities like temperature in turbulent fluid part 2. the case of large conductivity, Journal of Fluid Mechanics 5 (01) (1959) 134-139. doi:10.1017/S0022112059000106

[8] Z. Warhaft, Passive scalars in turbulent flows, Annual Review of Fluid Mechanics 32 (1) (2000) 203-240. doi:10.1146/annurev.fluid.32.1. 203.

[9] J. Schumacher, K. R. Sreenivasan, P. Yeung, Very fine structures in scalar mixing, Journal of Fluid Mechanics 531 (2005) 113-122. doi:10.1017/ S0022112005004040

[10] D. A. Donzis, P. K. Yeung, Resolution effects and scaling in numerical simulations of passive scalar mixing in turbulence, Physica D: Nonlinear n Phenomena 239 (14) (2010) 1278-1287. doi:10.1016/j.physd.2009.09. 024. 
[11] S. B. Pope, Small scales, many species and the manifold challenges of turbulent combustion, Proceedings of the Combustion Institute 34 (1) (2013) 1-31. doi:10.1016/j.proci.2012.09.009

[12] C. Flageul, S. Benhamadouche, É. Lamballais, D. Laurence, On the discontinuity of the dissipation rate associated with the temperature variance at the fluid-solid interface for cases with conjugate heat transfer, International Journal of Heat and Mass Transfer 111 (2017) 321-328. doi:10.1016/j.ijheatmasstransfer.2017.04.005

[13] N. Kasagi, Y. Tomita, A. Kuroda, Direct numerical simulation of the passive scalar field in a two-dimensional turbulent channel flow, in: Proceedings of the 1991 ASME JSME thermal engineering joint conference, 1991.

[14] H. Kawamura, K. Ohsaka, H. Abe, K. Yamamoto, Dns of turbulent heat transfer in channel flow with low to medium-high prandtl number fluid, International Journal of Heat and Fluid Flow 19 (5) (1998) 482-491. doi: 10.1016/S0142-727X (98) 10026-7.

[15] D. V. Papavassiliou, T. J. Hanratty, Transport of a passive scalar in a turbulent channel flow, International Journal of Heat and Mass Transfer 40 (6) (1997) 1303-1311. doi:10.1016/S0017-9310(96)00202-5.

[16] A. V. Johansson, P. M. Wikström, Dns and modelling of passive scalar transport in turbulent channel flow with a focus on scalar dissipation rate modelling, Flow, turbulence and combustion 63 (1) (2000) 223-245. doi: 10.1023/A: 1009948606944 .

[17] S. Gavrilakis, H. M. Tsai, P. R. Voke, D. C. Leslie, Direct and large eddy simulation of turbulence, notes on numerical fluid mechanics vol., U. Schumann, R. Friedrich, Vieweg, Braunschweig, DBR (1986) 105.

[18] K. Lam, S. Banerjee, Investigation of turbulent flow bounded by a wall and a free surface, Fundamentals of Gas-Liquid Flows 72 (1988) 29-38. 
[19] I. Tiselj, E. Pogrebnyak, C. Li, A. Mosyak, G. Hetsroni, Effect of wall boundary condition on scalar transfer in a fully developed turbulent flume, Physics of fluids 13 (4) (2001) 1028-1039. doi:10.1063/1.1350899.

[20] I. Tiselj, L. Cizelj, Dns of turbulent channel flow with conjugate heat transfer at prandtl number 0.01, Nuclear Engineering and Design 253 (2012) 153-160. doi:10.1016/j.nucengdes.2012.08.008.

[21] I. Tiselj, Tracking of large-scale structures in turbulent channel with direct numerical simulation of low prandtl number passive scalar, Physics of Fluids 26 (12) (2014) 125111. doi:10.1063/1.4905018

[22] J. Jiménez, P. Moin, The minimal flow unit in near-wall turbulence, Journal of Fluid Mechanics 225 (1991) 213-240. doi:10.1017/ S0022112091002033.

${ }_{425}$ [23] G. Brethouwer, J. C. R. Hunt, F. T. M. Nieuwstadt, Micro-structure and lagrangian statistics of the scalar field with a mean gradient in isotropic

口 turbulence, Journal of Fluid Mechanics 474 (2003) 193-225. doi:10.1017/ S0022112002002549.

[24] T. Gotoh, S. Hatanaka, H. Miura, Spectral compact difference hybrid computation of passive scalar in isotropic turbulence, Journal of Computational Physics 231 (21) (2012) 7398-7414. doi:10.1016/j.jcp.2012.07.010.

[25] T. A. Oliver, N. Malaya, R. Ulerich, R. D. Moser, Estimating uncertainties in statistics computed from direct numerical simulation, Physics of Fluids 26 (3) (2014) 035101. doi:10.1063/1.4866813.

${ }_{435}$ [26] P. Billingsley, Probability and measure, John Wiley \& Sons, 2008.

[27] S. Banerjee, R. Krahl, F. Durst, C. Zenger, Presentation of anisotropy properties of turbulence, invariants versus eigenvalue approaches, Journal of Turbulence (8) (2007) N32. doi:10.1080/14685240701506896. 
[28] J. L. Lumley, G. R. Newman, The return to isotropy of homogeneous 440 turbulence, Journal of Fluid Mechanics 82 (01) (1977) 161-178. doi: $10.1017 / \mathrm{S} 0022112077000585$ 